\title{
Characteristics of HBV infection in 705 HIV- infected patients under lamivudine-based antiretroviral treatment from three regions in China
}

This article was published in the following Dove Press journal:

Infection and Drug Resistance

Mingze Su,' Lingjie Liao, ${ }^{2} \mathrm{Hui}$ Xing, ${ }^{2}$ Shuai Wang, ${ }^{1,3}$ Yutang $\mathrm{Li}^{4}{ }^{4}$ Wei Lu, ${ }^{1,5}$ Lingyuan $\mathrm{He},{ }^{\prime}$ Juan Deng, 'Yiming Shao, ${ }^{2}$ Tong Li,' Hui Zhuang'

'Department of Microbiology and Infectious Disease Center, School of Basic Medical Sciences, Peking University Health Science Center, Beijing I00191, China; ${ }^{2}$ State Key Laboratory of Infectious Disease Prevention and Control, National Centre for AIDS/STD Control and Prevention, Chinese Centre for Disease Control and Prevention, Collaborative Innovation Centre for Diagnosis and Treatment of Infectious Diseases, Beijing I02206, China; ${ }^{3}$ Department of Laboratorial Science and Technology, School of Public Health, Peking University, Beijing I0019I, China; ${ }^{4}$ Key Laboratory of Medical Molecular Virology of Ministries of Health and Education, Shanghai Medical College, Fudan University, Shanghai 200032, China; ${ }^{5}$ Department of Laboratory, The Second Affiliated Hospital of Medical College of Xi'an Jiaotong University, Xi'an, Shaanxi 7I0004, China

Correspondence: Tong $\mathrm{Li}$

Department of Microbiology and Infectious Disease Center, School of Basic Medical Sciences, Peking University Health Science Center, 38 Xueyuan Road, Haidian District. Beijing 100191, China

Tel +861082802413

Fax +861082805654

Email tongli08@vip.sina.com

Hui Zhuang

Department of Microbiology and Infectious Disease Center, School of Basic Medical Sciences, Peking University Health Science Center, 38 Xueyuan Road, Haidian District, Beijing 100191 , China

Tel/Fax +861082801617

Email zhuangbmu@I26.com
Purpose: This study aimed to investigate the HIV and hepatitis B virus (HBV) co-infection in three HIV high endemic areas with different modes of HIV transmission and explore the HBV nucleos(t)ide analogue resistance (NUCr) substitutions in this cohort receiving antiretroviral therapy (ART).

Patients and methods: The enrolled $705 \mathrm{HIV}$-infected patients were from three different regions in China and received lamivudine-based ART for at least 1 year. After screening for hepatitis B surface antigen ( $\mathrm{HBsAg}$ ), the hepatitis B e antigen ( $\mathrm{HBeAg})$, and antibody against hepatitis B core antigen (anti-HBc and anti-HBc IgM), HBV DNA in plasma of patients positive for HBsAg was tested. The reverse transcriptase (RT) sequences of HBV were analyzed by direct sequencing.

Results: The overall HBsAg-positive rate was 7.1\% (50/705) (Guangxi [25/170, 14.7\%], Xinjiang [13/257, 5.1\%], and Henan [12/278, 4.3\%]). The age, transmission route, and ethnic status were found to be associated with HIV/HBV co-infection. We obtained $23 \mathrm{HBV}$ RT sequences belonging to genotypes $\mathrm{B}(9 / 23,39.1 \%), \mathrm{C}(13 / 23,56.5 \%)$, and $\mathrm{D}(1 / 23,4.4 \%)$. About $65.2 \%(15 / 23)$ of RT sequences harbored NUCr substitutions, all of which had combination substitution patterns. Patients with HBV NUCr had significantly higher HBV DNA level and ratio of $\mathrm{HBeAg}$-positive than those without NUCr. None of the patients was found to have both lamivudine-resistant HBV and HIV.

Conclusion: Our results suggested that $\mathrm{HBsAg}$-positive rate in the studied patients was similar to that of the general population in each of the studied regions, where the age, transmission route, and ethnic status might also play roles in HIV/HBV co-infection. The HBV combination NUCr substitutions were common in co-infected patients under ART. Monitoring of HBV infection and NUCr substitutions in HIV-infected patients would help in providing better clinical decisions and management, thus lowering patients' risks to develop end-stage liver diseases.

Keywords: human immunodeficiency virus, hepatitis B virus, co-infection, nucleos(t)ide analogue resistance, hepatitis B surface antigen

\section{Introduction}

Both HIV and hepatitis B virus (HBV) infections are serious public health problems worldwide. Globally, about 36.7 million people were living with HIV, and one million people died of HIV-related illness at the end of 2016 according to data from World Health Organization (WHO). ${ }^{1}$ Estimated 257 million people were infected with HBV and HBV-related complications led to about 884,000 deaths according to 2017 Global 
Hepatitis Report from WHO. ${ }^{2}$ Owing to the fact that these two viruses share similar transmission routes and risk factors of infection, HIV/HBV co-infection occurs. ${ }^{3}$ There were an estimated 2.7 million HIV/HBV co-infections among the 36.7 million (7.4\%) people living with HIV. ${ }^{2}$ China has the greatest number of HBV-infected people in the world, ${ }^{4}$ which may lead to a higher $\mathrm{HIV} / \mathrm{HBV}$ co-infection rate than the global average. A previous study had reported a $12 \% \mathrm{HIV} /$ HBV co-infection rate in China. ${ }^{5}$ In HIV/HBV co-infected patients, HIV infection could increase HBV replication that may accelerate the progression to liver fibrosis, liver cirrhosis, and hepatocellular carcinoma. Thus, liver disease-related morbidity and mortality have emerged as major threats in HIV/HBV co-infected patients. ${ }^{6}$ Consequently, providing effective management and antiviral therapy for HIV/HBV co-infected patients is highly significant.

Lamivudine (3TC) is one of the pioneer reverse transcriptase (RT) inhibitors used in antiretroviral therapy (ART). ${ }^{7}$ Due to its low price, good antiviral efficacy, and less adverse effects, it has been extensively used, especially in developing countries. Since both HIV and HBV undergo reverse transcription during replication, $3 \mathrm{TC}$ is also effective against $\mathrm{HBV}$, and it has been a first-line drug to treat patients with chronic HBV infection for many years. However, today most guidelines for the prevention and treatment of chronic HBV infection are not recommending $3 \mathrm{TC}$ as a first-line drug against HBV owing to its low genetic barrier to nucleos $(\mathrm{t})$ ide analogue resistance (NUCr) substitutions in $\mathrm{RT}^{8-10}$ A previous study suggested that HBV 3TC-resistant substitutions contributed to $\sim 20 \%-25 \%$ of annual substitution rate among $\mathrm{HIV} / \mathrm{HBV}$ co-infected individuals. ${ }^{11}$ The rtM204V/I is a well-known primary NUCr substitution leading to $\mathrm{HBV}$ resistance to 3TC and telbivudine, and providing a genetic background for entecavir (ETV) resistance development. Importantly, nowadays, ETV is one of the two recommended first-line drugs to treat chronic HBV infections. Therefore, the best choice for rtM204V/I harboring patients is tenofovir disoproxil fumarate (TDF)-based treatment. ${ }^{12}$ In addition, rtL80I/V, rtV173L, and rtL180M are the secondary resistance substitutions, which often coexist with rtM204V/I and could restore the impaired replication activity of rtM204V/I mutant. ${ }^{13,14}$

In China, National Free Antiretroviral Treatment Program recommended $3 \mathrm{TC}$ as the first-line ART regimen for HIV patients since 2008. The TDF-based combination ART was recommended for treatment of $\mathrm{HIV} / \mathrm{HBV}$ co-infected individuals in China since 2012. ${ }^{15,16}$ However, TDF/TDF +3 TC-based ART remains unavailable in some resourcelimited regions, and the 3TC-based ART has been continually in use. ${ }^{17}$ Some HIV-infected patients in China have received 3TC-based ART for almost 10 years. For those HIV/HBV co-infected patients receiving 3TC-based ART, they still have high risks to develop HBV-resistant substitutions to 3TC and end-stage liver diseases. From the public health point of view, they have potential importance in transmission of 3TCresistant $\mathrm{HBV}$ mutants to non-immunized or immunization failure HBV-uninfected persons.

In this study, we first investigated the detection rate of hepatitis B surface antigen (HBsAg) in a cohort of $705 \mathrm{HIV}$ infected patients receiving 3TC-based ART for at least 1 year. The patients were recruited from the northern, southern, and northwestern regions of China where HIV infection was highly endemic. Then, we studied HBV NUCr substitutions among HIV/HBV co-infected individuals. The results would help in patient management and provide basic information on HBV prevalence and characteristics for HIV-infected patients in China.

\section{Patients and methods \\ Patients and study design}

This study was approved by the Institutional Review Board at the National Center for AIDS/STD Control and Prevention of Chinese Center for Disease Control and Prevention (China CDC) with an approval number of X100517174 (May 17, 2010). The written informed consents were obtained from the patients. This cross-sectional study enrolled 705 HIV-infected patients diagnosed by CDC.

The studied population was from Henan province (Henan), Guangxi Zhuang autonomous region (Guangxi), and Xinjiang Uygur autonomous region (Xinjiang), which are located in the northern, southern, and northwestern regions of China, respectively. They belonged to HIV high endemic areas with different modes of HIV transmission reported by the National Health Commission of the People's Republic of China. All patients had received for at least 1 year 3TC-based ART that did not contain TDF or other drugs used to treat HBV infection. The patients did not have HBV screening at initiation of ART. The age, gender, HIV transmission route, ethnic and education level were according to patient's report in the questionnaire. The plasma samples were collected in 2010 and properly stored at $-80^{\circ} \mathrm{C}$ until use.

The flowchart in Figure 1 presents the study design.

\section{Laboratory tests for HIV}

CD4 cell count was detected by flow cytometry (FACSCalibur; BD, Franklin Lakes, NJ, USA) within 24 hours after plasma sample collection at provincial CDC laboratories. ${ }^{16}$ 


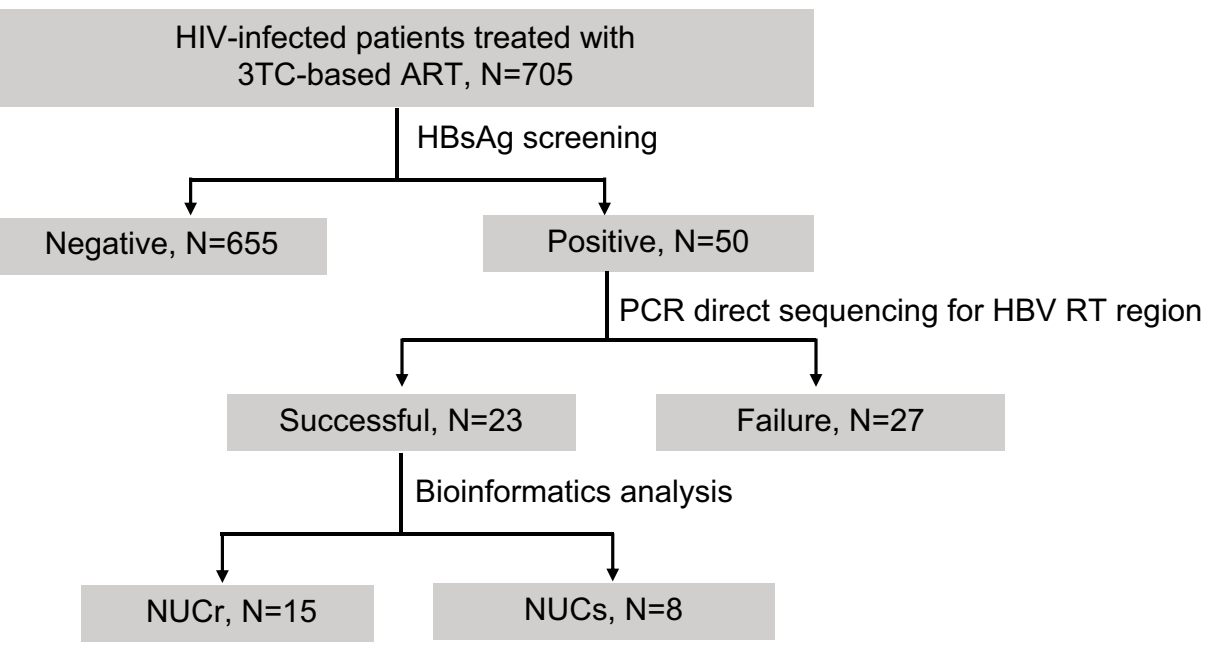

Figure I Flowchart of study design.

Abbreviations: ART, antiretroviral treatment; $\mathrm{HBsAg}$, hepatitis $B$ surface antigen; HBV, hepatitis $B$ virus; $N U C r$, nucleos $(t)$ ide analogue resistance; $N U C s$, nucleos(t)ide analogue susceptible; RT, reverse transcriptase; $3 T C$, lamivudine.

HIV RNA was quantified with real-time NASBA (NucliSens EasyQ; bioMerieux, Marcy l'Etoile, France) or COBAS (Roche Applied Biosystems, Mannheim, Germany) according to manufacturer's recommendations. For those with HIV RNA level $\geq 1,000$ copies/mL, HIV-1 partial polymerase genes (protease amino acid [AA] 1-99 and RT AA 1-252) were amplified and sequenced for drug resistance analysis. ${ }^{16}$

\section{Laboratory tests for HBV}

HBsAg, hepatitis B e antigen ( $\mathrm{HBeAg}$ ), antibody against hepatitis B core antigen (anti-HBc), and anti-HBc IgM were detected by corresponding commercial ELISA kits (Kehua, Shanghai, China). The condition where HIV patients were positive for HBsAg is defined as HIV/HBV co-infection in this study.

For co-infected patients, HBV DNA level was measured using quantitative PCR by employing HBV DNA Quantitative Fluorescence Diagnostic Kit (Sansure Biotech, Hunan, China). The lower limit of detection was $100 \mathrm{IU} / \mathrm{mL}$ (2 log IU/ $\mathrm{mL}$ ). The reactions were run in ABI StepOnePlus real-time PCR system (Applied Biosystems, Fostor City, CA, USA).

HBV DNA was extracted for RT amplification, sequencing, and drug resistance analysis. The methods followed were according to our previous study. ${ }^{18}$

\section{Sequence analyses}

HIV drug resistance analyses used the Stanford University HIV Drug Resistance Database (https://hivdb.stanford.edu/), ${ }^{16}$ which was also used for HBV NUCr analyses and genotyping. The 11 classical NUCr-related AA sites (rtL80, rtI169, rtV173, rtL180, rtA181, rtT184, rtA194, rtS202, rtM204, rtN236, rtM250) in HBV RT were carefully studied. ${ }^{19}$

\section{Statistical analyses}

Statistical analyses were performed by SPSS 20.0 (SPSS Inc., Chicago, IL, USA). Student's $t$-test, one-way ANOVA, or nonparametric test were used to examine the difference for continuous data, while chi-squared test or Fisher's exact test was applied for categorical data. Univariate- and multivariate-adjusted logistic regression models were used to estimate the odds ratio (OR) and the corresponding 95\% confidence interval (CI) for factors associated with HIV/ HBV co-infection. $P<0.05$ was considered as statistically significant.

\section{Results \\ The patients}

Overall characteristics of $705 \mathrm{HIV}$-infected patients are summarized in Table S1. In general, 39.4\% (278/705), 24.1\% (170/705), and $36.5 \%$ (257/705) of the patients were recruited from Henan, Guangxi, and Xinjiang, respectively. Henan patients were significantly older than those in the other two regions $(P<0.001)$.

The blood transfusion, heterosexual and intravenous transmissions were ranked as top three transmission routes and accounted for $96.6 \%(681 / 705)$ of the HIV infections. 
However, HIV transmission routes were significantly different in different regions $(P<0.001)$. The patients in Henan and Guangxi acquired HIV infection mainly through blood transmission $(269 / 278,96.8 \%)$ and heterosexual transmission $(141 / 170,82.9 \%)$, respectively; while those in Xinjiang were mainly by either intravenous $(128 / 257,49.8 \%)$ or heterosexual $(105 / 257,40.8 \%)$ transmission route. The CD4 cell count and HIV RNA detectable rate also showed regional significant differences $(P<0.001, P<0.001)$. The low overall HIV RNA detectable rate $(138 / 705,19.6 \%)$ might be due to ART.

\section{$\mathrm{HBsAg-positive} \mathrm{patients}$}

We found $7.1 \%(50 / 705)$ of the patients showed positive HBsAg, who were defined as having co-infection of HIV/ HBV. The positive rates of HBsAg varied in three regions with the highest in Guangxi $(25 / 170,14.7 \%)$, followed by $5.1 \%(13 / 257)$ in Xinjiang and 4.3\% (12/278) in Henan. In addition, 49 of 50 were positive for anti-HBc antibodies, one of whom had positive anti-HBc IgM. This patient was found to harbor NUCr substitutions and had a HBV DNA level of $6.6 \log \mathrm{IU} / \mathrm{mL}$. The data suggested that at least 48 of 50 patients had chronic HBV infection.

Basic characteristics of HIV/HBV co-infected patients are presented in Table 1. In multivariate analysis (Table 2), age $(\mathrm{OR}=0.96,95 \% \mathrm{CI}[0.93-1.00], P=0.045)$, intravenous $(\mathrm{OR}=2.91,95 \% \mathrm{CI}[1.01-8.36], P=0.048)$ and sexual (OR $=2.79,95 \% \mathrm{CI}[1.20-6.49], P=0.017)$ transmission routes, and ethnic status $(\mathrm{OR}=0.24,95 \% \mathrm{CI}[0.10-0.58], P=0.001)$ were independently associated with HIV/HBV co-infection. Older age and minorities compared with Han ethnic group were protective factors for HIV/HBV co-infection, while intravenous and sexual transmission routes compared with blood transmission were found to be risk factors for HIV/ HBV co-infection. Gender, education level, CD4 cell count, and HIV RNA were not found to be associated with HIV/ HBV co-infection.

\section{HBV sequence analyses}

Among $50 \mathrm{HIV} / \mathrm{HBV}$ co-infected patients, $23 \mathrm{HBV}$ RT sequences were successfully obtained (GenBank accession numbers: MH271235-MH271257). Failure in sequencing

Table I Overall characteristics of patients with HIV/HBV co-infection

\begin{tabular}{|c|c|c|c|c|c|}
\hline Characteristics & Overall $(\mathrm{N}=50)$ & Henan $(\mathbf{N}=12)$ & Guangxi ( $\mathbf{N}=\mathbf{2 5})$ & Xinjiang $(\mathrm{N}=13)$ & $P$-value ${ }^{a}$ \\
\hline Age (years), median (range) & $36(2 \mid-6 I)$ & $42(35-53)$ & $32(2 \mid-6 I)$ & $31(25-42)$ & 0.002 \\
\hline Gender, male/female (male\%) & $30 / 20(60.0)$ & $6 / 6(50.0)$ & $16 / 9(64.0)$ & $8 / 5(6 I .5)$ & 0.712 \\
\hline Transmission route, $\mathrm{N}(\%)$ & & & & & $<0.001$ \\
\hline Blood transmission & $12(24.0)$ & $12(100.0)$ & $0(0.0)$ & $0(0.0)$ & \\
\hline Intravenous & $12(24.0)$ & $0(0.0)$ & $6(24.0)$ & $6(46.2)$ & \\
\hline Sexual & $25(50.0)$ & $0(0.0)$ & $19(76.0)$ & $6(46.2)$ & \\
\hline Others/unknown & I (2.0) & $0(0.0)$ & $0(0.0)$ & I (7.7) & \\
\hline Ethnic, N (\%) & & & & & $<0.001$ \\
\hline Han & $39(64.3)$ & $12(100.0)$ & $24(96.0)$ & $3(2.3)$ & \\
\hline Minorities ${ }^{b}$ & $9(34.2)$ & $0(0.0)$ & $0(0.0)$ & $9(69.2)$ & \\
\hline Others/unknown & $2(1.6)$ & $0(0.0)$ & I (4.0) & $\mathrm{I}(7.7)$ & \\
\hline Education, N (\%) & & & & & 0.276 \\
\hline Primary school or below & $15(30.0)$ & $6(50.0)$ & $5(20.0)$ & $4(30.8)$ & \\
\hline Junior high school & $24(48.0)$ & $6(50.0)$ & $12(48.0)$ & $6(46.2)$ & \\
\hline Senior high school & $8(16.0)$ & $0(0.0)$ & $6(24.0)$ & $2(15.4)$ & \\
\hline College or higher & $\mathrm{I}(2.0)$ & $0(0.0)$ & $0(0.0)$ & I (7.7) & \\
\hline Unknown & $2(4.0)$ & $0(0.0)$ & $2(8.0)$ & $0(0.0)$ & \\
\hline CD4 cell count (cells $/ \mu \mathrm{L}$ ), median (range) & $353(114-1274)$ & $359(160-1274)$ & $323(114-1047)$ & $395(148-980)$ & 0.558 \\
\hline HIV RNA, det/undet (det\%)c & $7 / 43(14.0)$ & $3 / 9(25.0)$ & $0 / 25(0.0)$ & $4 / 9(30.8)$ & 0.016 \\
\hline HBV DNA (log IU/mL), median (range) ${ }^{d}$ & $2.6(2.0-8.1)$ & $4.9(2.0-8.1)$ & $2.4(2.0-6.8)$ & $2.6(2.0-6.7)$ & 0.034 \\
\hline Anti-HBc, pos/neg (pos\%) & $49 / 1(98.0)$ & $12 / 0(100.0)$ & $24 / 1(96)$ & $13 / 0(100)$ & 1.000 \\
\hline Anti-HBc IgM, pos/neg (pos\%) & $\mathrm{I} / 48(10.0)$ & $\mathrm{I} / \mathrm{I} \mathrm{I}(33.3)$ & $0 / 25(4.0)$ & $0 / 13(0.0)$ & 0.240 \\
\hline $\mathrm{HBeAg}$, pos/neg (pos\%) & $13 / 37(26.0)$ & $5 / 7(41.7)$ & $7 / 18(28.0)$ & $1 / 12(7.7)$ & 0.146 \\
\hline HBV genotype, $B / C / D$ & $9 / 13 / 1$ & $\mathrm{I} / \mathrm{I} \mathrm{I} / 0$ & $7 / 2 / 0$ & $1 / 0 / 1$ & 0.004 \\
\hline
\end{tabular}

Notes: ${ }^{a}$ The statistical analyses included the comparisons among the patient groups from three regions of China, respectively. ${ }^{\mathrm{b}} \mathrm{The}$ ethnic minority was Uygur. ${ }^{\mathrm{c}}$ The level of HIV RNA higher and lower than the lower limit of detection (LoD) was defined as detectable (det) and undetectable (undet), respectively. ${ }^{\mathrm{d}}$ The LoD values (2 log IU/mL) were used for the determination of HBV DNA levels in statistical analysis if the raw data reported were lower than LoD.

Abbreviations: anti- $\mathrm{HBc}$, antibody against hepatitis core antigen; $\mathrm{HBeAg}$, hepatitis $\mathrm{B}$ e antigen; $\mathrm{HBs} \mathrm{Ag}$, hepatitis $\mathrm{B}$ surface antigen; $\mathrm{HBV}$, hepatitis $\mathrm{B}$ virus. 
Table 2 Variables associated with HIV/HBV co-infection analyzed by univariate and multivariate logistic regression analysis ${ }^{\mathrm{a}}$

\begin{tabular}{|c|c|c|c|c|}
\hline \multirow[t]{2}{*}{ Characteristics } & \multicolumn{2}{|l|}{ Univariate } & \multicolumn{2}{|l|}{ Multivariate } \\
\hline & Crude OR (95\% Cl) & $P$-value & Adjusted OR (95\% Cl) & $P$-value \\
\hline Age (years) & $0.97(0.93-1.00)^{*}$ & $0.040 *$ & $0.96(0.93-1.00)^{*}$ & $0.045^{*}$ \\
\hline \multicolumn{5}{|l|}{ Gender, male/female } \\
\hline Male & 1.00 & & 1.00 & \\
\hline Female & $1.07(0.59-1.92)$ & 0.833 & $0.99(0.49-2.03)$ & 0.985 \\
\hline \multicolumn{5}{|l|}{ Transmission route } \\
\hline Blood transmission & 1.00 & & 1.00 & \\
\hline Intravenous & $1.83(0.80-4.17)$ & 0.153 & $2.91(1.01-8.36)^{*}$ & $0.048^{*}$ \\
\hline Sexual & $2.36(1.16-4.81)^{*}$ & $0.018^{*}$ & $2.79(1.20-6.49)^{*}$ & $0.017^{*}$ \\
\hline \multicolumn{5}{|l|}{ Ethnic } \\
\hline Han & 1.00 & & 1.00 & \\
\hline Minorities & $0.49(0.25-0.97)^{*}$ & $0.04 I^{*}$ & $0.24(0.10-0.58)^{*}$ & $0.00 I^{* *}$ \\
\hline \multicolumn{5}{|l|}{ Education } \\
\hline Primary school or below & 1.00 & & 1.00 & \\
\hline Junior high school & $1.59(0.82-3.10)$ & 0.170 & $1.32(0.66-2.63)$ & 0.435 \\
\hline Senior high school & $1.82(0.74-4.44)$ & 0.190 & $1.34(0.52-3.47)$ & 0.542 \\
\hline College or higher & $1.31(0.16-10.67)$ & 0.798 & $0.91(0.11-7.83)$ & 0.929 \\
\hline CD4 cell count (cells/ $\mu \mathrm{L})$ & $1.00(1.00-1.00)$ & 0.497 & $1.00(1.00-1.00)$ & 0.968 \\
\hline \multicolumn{5}{|l|}{ HIV RNA, det/undet } \\
\hline det & 1.00 & & 1.00 & \\
\hline undet & $0.65(0.29-1.48)$ & 0.306 & $1.14(0.45-2.89)$ & 0.779 \\
\hline
\end{tabular}

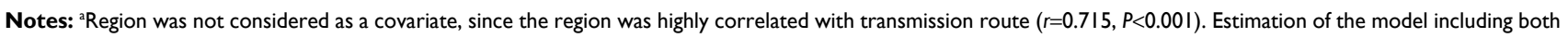
region and transmission route was not precise for the effect of collinearity. However, the results from the model not including region were similar to the results from the model excluding transmission route. ${ }^{b}$ The level of HIV RNA higher and lower than the lower limit of detection (LoD) was defined as detectable (det) and undetectable(undet), respectively. $* P<0.05, * * P<0.01$.

Abbreviations: HBV, hepatitis B virus.

mainly occurred at amplification step. This might be due to the low HBV DNA levels compared with those in succeeded ones (median $[\log \mathrm{IU} / \mathrm{mL}]: 2.0$ vs $4.6, P<0.001$ ).

Three HBV genotypes were found, which were $39.1 \%$ (9/23) genotype B, 56.5\% C (13/23), and 4.4\% (1/23) D (Table 1). The HBV genotypes in the three studied regions of China displayed known geographic distribution patterns. ${ }^{20}$

About $65.2 \%$ (15/23) of HBV RT sequences were found to harbor NUCr substitutions. All (15/15, 100.0\%) NUCr RT sequences had rtM204V/I substitution. Based on the presence or absence of NUCr substitutions, the patients were grouped into NUCr subgroup ( $\mathrm{N}=15)$ and NUC susceptible (NUCs, $\mathrm{N}=8$ ) subgroup, respectively. The comparison between the two subgroups is shown in Table S2. The age, sex ratio, therapy period, and HBV genotypes exhibited no significant statistical differences between the subgroups. However, both the HBV DNA level (median [range] log IU/mL: 5.9 [2.2-8.1] vs 2.1 [2.0-7.9], $P=0.005$ ) and $\mathrm{HBeAg}$-positive rate (pos/neg [pos\%]: $10 / 5$ [66.7\%] vs 1/7 [12.5\%], $P=0.027$ ) were found to be significantly higher in the NUCr subgroup than those in NUCs subgroup (Table S2). Interestingly, although CD4 counts showed no significant difference between subgroups, the HIV RNA was detectable only in NUCs subgroup (4/8, $50.0 \%$ ), in which two had HIV drug-resistant substitutions to nucleoside and/or non-nucleoside RT inhibitors. The drug-resistant HIV might replicate well and contribute to positive HIV RNA.

\section{HBV NUCr substitution analyses}

Among the 11 studied classical NUCr-associated AA sites, four sites were found to have substitutions. They were $\mathrm{rtL} 80 \mathrm{I} / \mathrm{V} / \mathrm{F}, \mathrm{rtV} 173 \mathrm{~L}, \mathrm{rtL} 180 \mathrm{M}$, and rtM204V/I. The overall substitution detection rate was $14.6 \%$ ([AA sites with substitutions/total AA sites studied in 23 patients $] \times 100 \%=37 /$ $[11 \times 23] \times 100 \%)$. The substitution detection rate of $\mathrm{rtL} 80 \mathrm{I} / \mathrm{F}$, rtV173L, rtL180M, and rtM204V/I was 21.7\% (5/23), 17.4\% (4/23), 56.5\% (10/23), and 65.2\% (15/23), respectively (Figure $2 \mathrm{~A}$ ). In addition, there were four combination substitution patterns of NUCr, in which rtL180M+rtM204V/I accounted for $40.0 \%$ (6/15) (Figure 2B).

Furthermore, we investigated HIV resistance to nucleos(t) ide analogues (NUC) in these 23 patients with available HBV RT sequences. The results revealed that 7 patients carried 3TC-susceptible HIV and HBV, 15 had 3TC-resistant HBV, 
A

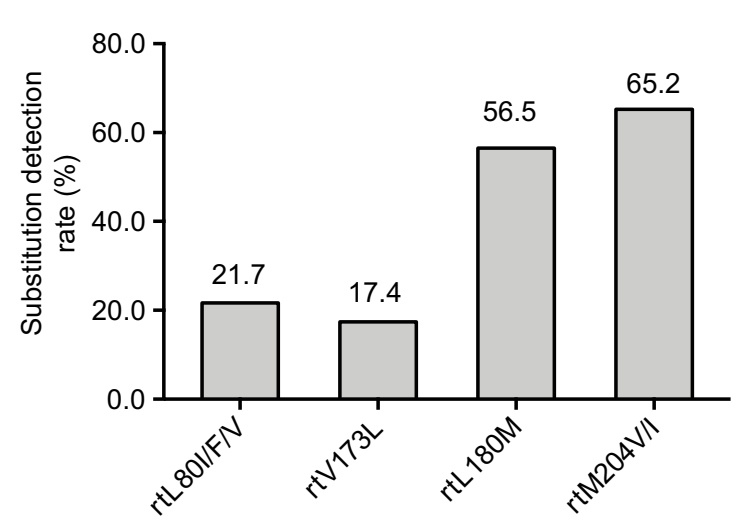

B

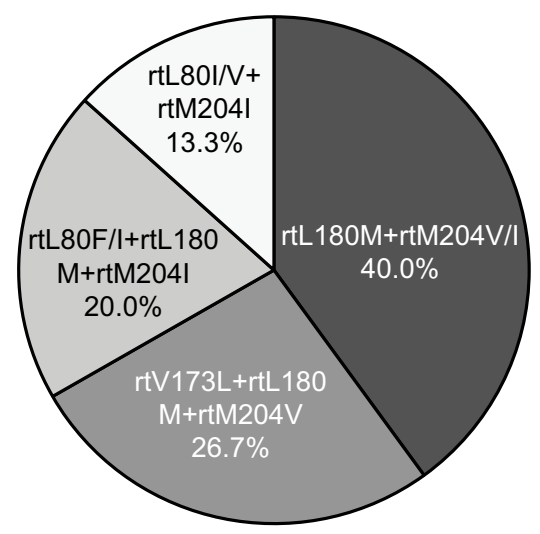

Figure 2 HBV NUCr substitutions. (A) Substitution detection rate of NUCr-associated AA sites. (B) Proportion of combination substitutions. Abbreviations: AA, amino acid; HBV, hepatitis $B$ virus; $N U C r$, nucleos(t)ide analogue resistance; $R T$, reverse transcriptase.

and 1 had 3TC-resistant HIV. None was found to have both 3TC-resistant HBV and HIV.

\section{The influence of therapy duration}

The median ART duration for HIV/HBV co-infected patients was 3.0 years (range: 1.0-6.4 years). We did not find any significant differences in CD4 cell counts and HIV RNA levels between patients receiving ART less than and longer than 3.0 years. However, the HBV DNA level in patients with ART less than 3.0 years was significantly lower than that of patients with ART longer than 3.0 years (median: $2.0 \log$ IU/ $\mathrm{mL}$ vs $3.3 \log \mathrm{IU} / \mathrm{mL}, P=0.027$ ).

\section{Discussion}

In this study, we found a total HBsAg-positive detection rate of $7.1 \%$, which was lower than $12 \% \mathrm{HIV} / \mathrm{HBV}$ coinfection rate in China reported by a previous study. ${ }^{5}$ This might be largely due to the fact that $36 \%$ of $(254 / 705)$ the studied population were blood donors, who were screened for HBsAg before donating blood and normally had very low HBsAg-positive rate. ${ }^{21}$ Our data showed that only 11 of them $(11 / 254,4.3 \%)$ were detected with positive HBsAg. In addition, the prevalence of HBsAg in the 15-59-year general population of Henan, Guangxi, and Xinjiang was reported as $5.3 \%, 12.4 \%$, and $3.9 \%$, respectively, by a 2006 study. ${ }^{22}$ Similar variances in HBsAg-positive rates among the three studied regions were also found in our study $(4.3 \%, 14.7 \%$, and $5.1 \%$ in Henan, Guangxi, and Xinjiang), suggesting the regional epidemiological background of $\mathrm{HBV}$ infection might also affect HIV/HBV co-infection rate.

It can be noticed that our patients received 3TC-based ART. However, this might not largely affect HBsAg detection rate. It is known that $3 \mathrm{TC}$ and other NUC used to treat chronic HBV infection have difficulties in reducing the serum HBsAg to an undetectable level. Chang et al reported that only $1.4 \%(2 / 145)$ lost HBsAg within 5 years of ETV treatment. ${ }^{23}$ The HBsAg-positive rate that we revealed in this study might not be affected by ART. In addition, China launched national HBV immunization strategy to new born babies since $1992 .{ }^{24}$ The patients in this study had a median age of 37 years and only one was born after 1992. Thus, the HBsAg-positive rate in our study was hardly affected by the immunization protection either.

Moreover, HIV was firstly transmitted to mainland China in $1985 .{ }^{25}$ From the late 1980 s to the early 1990 s, commercial plasma donation was popular in some rural areas of Henan province. It led to HIV infection of many commercial plasma donors through illegal blood collection practices without adequate screening and sterilization procedures of blood donation in Henan. In addition, the epidemic had spread rapidly due to intravenous drug injection and sexual behaviors in Guangxi and Xinjiang since 1995. ${ }^{26}$ So the patients with HIV infection in Henan were significantly older than those in the other two areas. According to a CDC report, the majority (86.35\%) of HIV-infected cases were found in people aged 20-50 years, with $32.38 \%$ of cases among those aged 30-39 
years during 2004-2013. ${ }^{27}$ Therefore, the patients in Henan might be infected with HIV earlier as compared with the national average.

The mother-to-infant transmission has long been known to be the major transmission route for $\mathrm{HBV}$ infection in China. ${ }^{28}$ Of the $50 \mathrm{HIV} / \mathrm{HBV}$ co-infected patients, 11 were blood donors. HBV vertical transmission might be ruled out for them, because they should have been tested for serum HBsAg-negative before blood donation. For the rest of the 39 patients, 97.4\% (38/39) had reported possible routes of HIV infection. Multivariate analysis results revealed intravenous drug injection and sexual behaviors were risk factors for HIV/HBV co-infection compared with blood transmission route. Intravenous drug injection and heterosexual behaviors accounted for $11(11 / 155,7.1 \%)$ and $26(26 / 253,10.3 \%)$ cases, respectively. They had high risks of HBV horizontal transmission, although vertical transmission might not be completely ruled out. The HIV and HBV infections might simultaneously occur (co-infection) or can be acquired by super-infection. HBV immunizations to the new born babies are applied nationwide in China nowadays, which would help to reduce HBV mono-infection and HIV/HBV co-infection/ super-infection in the near future.

Studies on the general population by Wang et al and Zeng et al, revealed that HBV genotypes $\mathrm{B}$ and $\mathrm{C}$ were predominant genotypes in the southern and northern China, respectively, while genotype D was mostly found in the northwest of China. ${ }^{29,30}$ In our study, $77.8 \%$ (7/9) of cases with genotype B, 84.6\% (11/13) with genotype C, and 100.0\% (1/1) with genotype D were found in sequences from Guangxi (southern China), Henan (northern China), and Xinjiang (northwestern China) patients, respectively. The geographic distribution patterns of HBV genotype in the HIV/HBV co-infected patients resembled those found in the general population. The results suggested that HIV/HBV co-infection might not have HBV genotype bias.

Generally, ART duration would impact the virological response (including HIV and $\mathrm{HBV}$ ) and host immune status, which is reflected by the CD4 cell count. We found that the HBV DNA level in patients with ART less than 3.0 years was significantly lower than that of patients with ART longer than 3.0 years. This result was similar to those from Pal et al's study, ${ }^{17}$ which showed that the HIV/HBV co-infected patients with longer ART duration had a higher HBV DNA level. It is known that HBV NUCr substitutions increase along with the increased antiviral drug duration and result in increased HBV DNA levels. The cumulative incidence of 3 TC-resistant substitutions was 24\%, 38\%, 49\%, 67\%, and
$70 \%$ at $1,2,3,4$, and 5 years of treatment, respectively. ${ }^{31}$ In our study, $65.2 \%(15 / 23)$ of NUCr substitutions occurred among the 23 cases with HBV sequences, and the average ART duration was 3.7 years. Our finding was similar to $67 \%$ of 3TC-resistant substitution rate at 4 years of treatment mentioned previously. Although this cross-sectional study could not rule out the possibility of transmission of $\mathrm{NUCr}$ HBV strains, the others and our previous studies have shown that the frequency of appearance of NUCr substitutions was very low before receiving antiviral therapy, ${ }^{12,13,32}$ suggesting that our patients might have little chance to develop 3TCresistant HBV in the absence of NUC therapy. Actually, the previously reported 3TC-resistant substitutions have been largely attributed to NUC-induced substitutions. ${ }^{31}$

All $(15 / 15)$ the identified NUCr HBV sequences had rtM204V/I substitutions and were accompanied with one or two secondary NUCr substitution(s). Four patterns of 3TC-resistant substitutions were found (Figure 2). Although $15 \mathrm{NUCr}$ and 8 NUCs harboring patients had no significant difference in ART durations, the NUCr subgroup had significantly higher HBV DNA levels than those of NUCs subgroup (Table S1). Further, those with triple 3TC-resistant substitutions $(\mathrm{N}=7)$ had significantly higher HBV DNA levels than those with double 3TC-resistant substitutions $(\mathrm{N}=8)$ (median [range] log IU/mL: 6.8 [4.6-8.1] vs 4.7 (2.2-6.1), $P=0.004)$. Our results suggested that the secondary NUCr substitutions rescued the defective replication of $\mathrm{rtM} 204 \mathrm{~V} / \mathrm{I}$ mutants in HIV/HBV co-infected hosts, which have been previously reported in HBV mono-infected patients. In addition, all the NUCr substitution patterns identified in this study would increase the risk of emergence of ETV-resistant substitutions. TDF would be the only good choice left for these patients to prevent the progression to more severe liver diseases. Moreover, their roles as sources of HIV and drug-resistant HBV transmissions need to be alerted since there are still patients receiving 3TC-based ART today. Fortunately, we did not identify any case carrying both HBV and HIV 3TCresistant substitutions in this study, though the antiviral target of 3TC was shared by these two viruses. To screen HBV infection at initiation of ART among HIV-infected patients is important and is recommended nowadays. However, HIV provider's adherence to $\mathrm{HBV}$ screening recommendations in the established guidelines was lower when compared to hepatologists. ${ }^{33}$

Among $50 \mathrm{HIV} / \mathrm{HBV}$ co-infected patients, the detectable rate of HBV DNA $(62.0 \%, 31 / 50)$ was significantly higher than that of HIV RNA $(14.0 \%, 7 / 50)(P<0.001)$. ART is specifically designed for combating HIV infection, which has 
multiple targets on HIV replication. However, the only effective agent of ART against HBV in this study is 3TC, which has relatively lower antiviral potency and low genetic barrier for the emergence of NUCr. Although 3TC dose in ART for HIV/HBV co-infected patients $(300 \mathrm{mg} / \mathrm{d})$ was higher than that for HBV mono-infected patients (100 mg/d), NUCr combination substitutions occurred in 15 studied patients. Today's guidelines recommend that ART should contain TDF for HIV/HBV co-infected patients. ${ }^{34,35}$

Although there were important findings, the limitations existed. One was the unavailability of serum alanine and aspartate transaminase levels, which could indicate the extent of damage to hepatocytes and thus liver disease progression. Another limitation was that this was a cross-sectional study. It was not possible to trace back the HBsAg status at the initiation of ART, and to follow the long-term clinical outcome of ART on the HIV mono-infected and HIV/HBV co-infected ones.

\section{Conclusion}

Our results suggested that HBsAg-positive rate in the studied patients was similar to that of the general population in each of the studied regions. The age, transmission route, and ethnic status might be associated with HIV/HBV co-infection. For HIV/HBV co-infected patients, the detection rate of 3TCresistant substitutions was in line with those having $\mathrm{HBV}$ mono-infection, and the HBV combination NUCr substitutions were very common. It is important to monitor HBV NUCr substitutions in the co-infected population on ART along with HIV drug resistance surveillance. This would provide a better clinical decision and management for those with HIV/HBV co-infection and to lower patients' risks to develop end-stage liver diseases.

\section{Acknowledgments}

This study was supported by the financial grants from Major Science and Technology Special Project of China Thirteenth Five-year Plans (2017Z X 10202202-004-004 and 2018Z X 10731101-001-004) and National Natural Science Foundation of China (No 81772174).

\section{Author contributions}

Mingze Su performed experiments, analyzed data and drafted the manuscript; Lingjie Liao and Hui Xing collected clinical samples and patients' information; Shuai Wang, Yutang Li, Wei Lu, Lingyuan He and Juan Deng participated in experiments and database set up; Yiming Shao, Tong Li, and Hui Zhuang designed the study, actively involved in data analyses and revised the manuscript. All authors contributed toward data analysis, drafting and critically revising the paper and agree to be accountable for all aspects of the work.

\section{Disclosure}

The authors report no conflicts of interest in this work.

\section{References}

1. World Health Organization. Global health sector strategy on HIV, 2016-2021. Available from: http://www.who.int/mediacentre/factsheets/ fs360/en/. Accessed August 17, 2018.

2. World Health Organization. Global hepatitis report, 2017. 2017. Available from: http://www.who.int/hepatitis/publications/global-hepatitisreport2017/en/. Accessed August 17, 2018.

3. Prussing C, Chan C, Pinchoff J, et al. HIV and viral hepatitis co-infection in New York City, 2000-2010: prevalence and case characteristics. Epidemiol Infect. 2015;143(7):1408-1416.

4. Polaris Observatory Collaborators. Global prevalence, treatment, and prevention of hepatitis B virus infection in 2016: a modelling study. Lancet Gastroenterol Hepatol. 2018;3(6):383-403.

5. Zhang F, Zhu H, Wu Y, et al. HIV, hepatitis B virus, and hepatitis C virus co-infection in patients in the China National Free Antiretroviral Treatment Program, 2010-12: a retrospective observational cohort study. Lancet Infect Dis. 2014;14(11):1065-1072.

6. Thio CL. Hepatitis B and human immunodeficiency virus coinfection. Hepatology. 2009;49(5 Suppl):S138-S145.

7. Montaner JS, Lima VD, Harrigan PR, et al. Expansion of HAART coverage is associated with sustained decreases in HIV/AIDS morbidity, mortality and HIV transmission: the "HIV Treatment as Prevention" experience in a Canadian setting. PLoS One. 2014;9(2):e87872.

8. European Association for the Study of the Liver. EASL 2017 Clinical Practice Guidelines on the management of hepatitis B virus infection. J Hepatol. 2017;67(2):370-398.

9. Sarin SK, Kumar M, Lau GK, et al. Asian-Pacific clinical practice guidelines on the management of hepatitis B: a 2015 update. Hepatol Int. 2016;10(1):1-98.

10. Terrault NA, Bzowej NH, Chang KM, et al. AASLD guidelines for treatment of chronic hepatitis B. Hepatology. 2016;63(1):261-283.

11. Li Y, Xie J, Han Y, et al. Lamivudine monotherapy-based cART is efficacious for HBV treatment in HIV/HBV coinfection when baseline HBV DNA <20,000 IU/mL. J Acquir Immune Defic Syndr. 2016;72(1): $39-45$.

12. Xu X, Xiang K, Su M, et al. HBV drug resistance substitutions existed before the clinical approval of nucleos(t)ide analogues: a bioinformatic analysis by GenBank data mining. Viruses. 2017;9(8).

13. Liu BM, Li T, Xu J, et al. Characterization of potential antiviral resistance mutations in hepatitis B virus reverse transcriptase sequences in treatment-naïve Chinese patients. Antiviral Res. 2010;85(3): 512-519.

14. Lok AS, Zoulim F, Locarnini S, et al. Antiviral drug-resistant HBV: standardization of nomenclature and assays and recommendations for management. Hepatology. 2007;46(1):254-265.

15. Li T, Guo F, LiY, et al. An antiretroviral regimen containing 6 months of stavudine followed by long-term zidovudine for first-line HIV therapy is optimal in resource-limited settings: a prospective, multicenter study in China. Chin Med J. 2014;127(1):59-65.

16. Kan W, Teng T, Liang S, et al. Predictors of HIV virological failure and drug resistance in Chinese patients after 48 months of antiretroviral treatment, 2008-2012: a prospective cohort study. BMJ Open. 2017;7(9):e16012.

17. Pal A, Sarkar N, Saha D, et al. High incidence of lamivudine-resistanceassociated vaccine-escape HBV mutants among HIV-coinfected patients on prolonged antiretroviral therapy. Antivir Ther. 2015;20(5):545-554.

18. Yang JX, Liu BM, Li XG, et al. Profile of HBV antiviral resistance mutations with distinct evolutionary pathways against nucleoside/nucleotide analogue treatment among Chinese chronic hepatitis B patients. Antivir Ther. 2010;15(8):1171-1178. 
19. Su M, Xiang K, Li Y, et al. Higher detection rates of amino acid substitutions in $\mathrm{HBV}$ reverse transcriptase/surface protein overlapping sequence is correlated with lower serum HBV DNA and HBsAg levels in $\mathrm{HBeAg}$-positive chronic hepatitis B patients with subgenotype B2. Infect Genet Evol. 2016;40:275-281.

20. Wang Z, Huang Y, Wen S, Zhou B, Hou J. Hepatitis B virus genotypes and subgenotypes in China. Hepatol Res. 2007;37(s1):S36-S41.

21. Zhang X, Tang H, Luo X, Chen H. Study on the serological status of HCV and HBV among HIV infected blood donors. Chin J AIDS STD 2002;8(4):229-230.

22. Cui F, Gong X, Chen Y, Wang F, Wei Z. Vaccination progress of hepatitis $B$ vaccine and epidemiology changes of carrying rate of hepatitis $B$ surface antigen by province in China, 1992-2006. Chin J Vacc Immun 2012;18(1):6-13.

23. Chang TT, Lai CL, Kew Yoon S, et al. Entecavir treatment for up to 5 years in patients with hepatitis B e antigen-positive chronic hepatitis B. Hepatology. 2010;51(2):422-430.

24. Liang X, Bi S, Yang W, et al. Evaluation of the impact of hepatitis B vaccination among children born during 1992-2005 in China. J Infect Dis. 2009;200(1):39-47.

25. Lu L, Jia M, Ma Y, et al. The changing face of HIV in China. Nature. 2008;455(7213):609-611.

26. Wu Z, Rou K, Cui H. The HIV/AIDS epidemic in China: history, current strategies and future challenges. AIDS Educ Prev. 2004;16(3 Suppl A):7-17.
27. Xu Y, Yang G, Liu H, et al. Epidemiologic features of AIDS in China, 2004-2013. Clin Infect Dis. 2015;60(1):167-169.

28. Yao JL. Perinatal transmission of hepatitis B virus infection and vaccination in China. Gut. 1996;38(Suppl 2):S37-S38.

29. Wang Z, Tanaka Y, Huang Y, et al. Clinical and virological characteristics of hepatitis $\mathrm{B}$ virus subgenotypes $\mathrm{Ba}, \mathrm{C} 1$, and $\mathrm{C} 2$ in China. J Clin Microbiol. 2007;45(5):1491-1496.

30. Zeng G, Wang Z, Wen S, et al. Geographic distribution, virologic and clinical characteristics of hepatitis B virus genotypes in China. J Viral Hepat. 2005;12(6):609-617.

31. Lim YS. Management of antiviral resistance in chronic hepatitis B. Gut Liver. 2017;11(2):189-195.

32. Han Y, Huang LH, Liu CM, et al. Characterization of hepatitis B virus reverse transcriptase sequences in Chinese treatment naive patients. $J$ Gastroenterol Hepatol. 2009;24(8):1417-1423.

33. Hearn B, Chasan R, Bichoupan K, et al. Low adherence of HIV providers to practice guidelines for hepatocellular carcinoma screening in HIV/ hepatitis B coinfection. Clin Infect Dis. 2015;61(11):1742-1748.

34. Ryom L, Boesecke C, Bracchi M, et al. Highlights of the 2017 European AIDS Clinical Society (EACS) Guidelines for the treatment of adult HIV-positive persons version 9.0. HIV Med. 2018;19(5):309-315.

35. Oh JM, Kyun J, Cho SW. Long-term lamivudine therapy for chronic hepatitis B in patients with and without cirrhosis. Pharmacotherapy. 2002;22(10):1226-1234. 


\section{Supplementary materials}

Table SI Overall characteristics of 705 HIV-infected patients

\begin{tabular}{|c|c|c|c|c|}
\hline Characteristics & Overall $(\mathrm{N}=705)$ & Henan $(\mathbf{N}=278)$ & Guangxi $(N=170)$ & Xinjiang $(\mathrm{N}=257)$ \\
\hline Age (years), median (range) & $37(10-78)$ & $43(10-68)$ & $34(18-78)$ & $34(19-65)$ \\
\hline Gender, male/female (male\%) & $4 \mid 3 / 292(58.6)$ & I64/II 4 (59.0) & $102 / 68(60.0)$ & $147 / \mid 10(57.2)$ \\
\hline \multicolumn{5}{|l|}{ Transmission route, $\mathrm{N}(\%)$} \\
\hline Blood & $273(38.7)$ & $269(96.8)$ & $\mathrm{I}(0.6)$ & $3(1.2)$ \\
\hline Intravenous & $155(22.0)$ & $0(0.0)$ & $27(15.9)$ & $128(49.8)$ \\
\hline Homosexual & $2(0.3)$ & $0(0.0)$ & $0(0.0)$ & $2(0.8)$ \\
\hline Heterosexual & $253(35.9)$ & $7(2.5)$ & $|4|(82.9)$ & $105(40.8)$ \\
\hline Others/unknown & $22(3.1)$ & $2(0.7)$ & $\mathrm{I}(0.6)$ & $19(7.4)$ \\
\hline \multicolumn{5}{|l|}{ Ethnic, N (\%) } \\
\hline Han & $453(64.3)$ & $277(99.6)$ & $157(92.4)$ & $19(7.4)$ \\
\hline Minorities $^{a}$ & $24 I(34.2)$ & I (0.4) & $5(2.9)$ & $235(91.4)$ \\
\hline Others/unknown & II (I.6) & $0(0.0)$ & $8(4.7)$ & $3(1.2)$ \\
\hline \multicolumn{5}{|l|}{ Education, N (\%) } \\
\hline Primary school or below & $291(4 I .3)$ & $150(54.0)$ & $53(31.2)$ & $88(34.2)$ \\
\hline Junior high school & $301(42.7)$ & II4 (4I.0) & $78(45.9)$ & $109(42.4)$ \\
\hline Senior high school & $89(12.6)$ & $13(4.7)$ & $27(15.9)$ & $49(19.1)$ \\
\hline College or higher & $15(2.1)$ & $\mathrm{I}(0.3)$ & $4(2.4)$ & $10(3.9)$ \\
\hline Unknown & $9(1.3)$ & $0(0.0)$ & $8(4.7)$ & $\mathrm{I}(0.4)$ \\
\hline CD4 cell count (cells $/ \mu \mathrm{L})$, median (range) & $395(39-2465)$ & $455(86-2465)$ & $322(55-1292)$ & $395(39-1220)$ \\
\hline HIV RNA, det/undet (det\%) ${ }^{\mathrm{b}}$ & 138/567 (19.6) & $45 / 233(16.2)$ & $1 / 169(0.6)$ & $92 / 165(35.8)$ \\
\hline
\end{tabular}

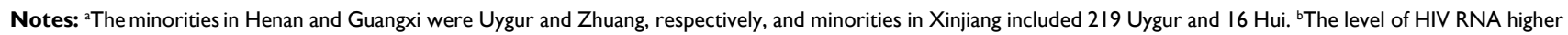
and less than the lower limit of detection was defined as detectable (det) and undetectable (undet), respectively.

Table S2 Characteristics of the HIV/HBV co-infected patients in NUCr and NUCs subgroups

\begin{tabular}{|c|c|c|c|}
\hline Characteristics & $\operatorname{NuCr}(\mathrm{N}=15)$ & NUCs $(\mathbf{N}=8)$ & $P$-value \\
\hline Age (years), median (range) & $38(2 I-59)$ & $37(3 I-53)$ & 0.560 \\
\hline Gender, male/female (male\%) & $7 / 8(46.7)$ & $4 / 4(50.0)$ & $>0.999$ \\
\hline Therapy period (years), median (range) & $3.7(1.0-6.4)$ & $4.4(2.5-6.1)$ & 0.229 \\
\hline CD4 cell count (cells/ $/ \mathrm{L})$, median (range) & $440(160-1274)$ & $319(175-583)$ & 0.357 \\
\hline HIV RNA, det/undet (det\%) ${ }^{\mathrm{a}}$ & $0 / 15(0.0)$ & $4 / 4(50.0)$ & 0.008 \\
\hline HBV DNA (log IU/mL), median (range) ${ }^{\mathrm{b}}$ & $5.9(2.2-8.1)$ & $2.1(2.0-7.9)$ & 0.005 \\
\hline $\mathrm{HBeAg}$, pos/neg (pos\%) & $10 / 5(66.7)$ & $\mathrm{I} / 7(12.5)$ & 0.027 \\
\hline HBV genotype, $B / C / D$ & $8 / 7 / 0$ & $|/ 6 /|$ & 0.089 \\
\hline
\end{tabular}

Notes: ${ }^{\top}$ The level of HIV RNA higher and lower than the lower limit of detection (LoD) was defined as detectable (det) and undetectable (undet), respectively. ${ }^{\mathrm{b} T h e} \mathrm{LoD}$ values $(2 \log \mathrm{IU} / \mathrm{mL}$ ) were used for the determination of HBV DNA levels in statistical analysis if the raw data were reported as lower than LoD.

Abbreviations: $\mathrm{HBeAg}$, hepatitis $B$ e antigen; $\mathrm{HBV}$, hepatitis $B$ virus; $N U C r$, nucleos $(\mathrm{t})$ ide analogue resistance; $N U C s$, nucleos $(\mathrm{t})$ ide analogue susceptible.

\section{Publish your work in this journal}

Infection and Drug Resistance is an international, peer-reviewed openaccess journal that focuses on the optimal treatment of infection (bacterial, fungal and viral) and the development and institution of preventive strategies to minimize the development and spread of resistance. The journal is specifically concerned with the epidemiology of antibiotic
Dovepress

resistance and the mechanisms of resistance development and diffusion in both hospitals and the community. The manuscript management system is completely online and includes a very quick and fair peerreview system, which is all easy to use. Visit http://www.dovepress.com/ testimonials.php to read real quotes from published authors. 